\title{
Mitglieder im Lockdown
}

\section{Eine Momentaufnahme}

Marianne Roth

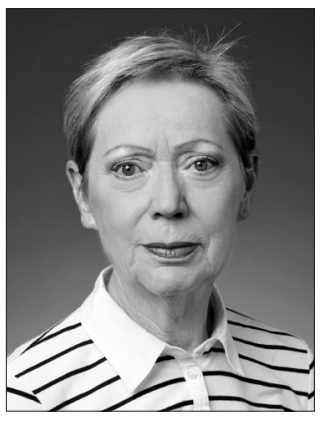

Die COVID-19-Pandemie hat bei vielen unserer Mitglieder grosse Unruhe und Besorgnis ausgelöst. Auch wenn wir versucht haben, ihnen die laufenden Entscheide des Bundesrats möglichst präzise zu vermitteln, blieben offene Fragen und Ungewissheiten, was dies für Auswirkungen auf die zukünftige Praxis haben würde. Für uns als Berufsverband war wichtig, von unseren Mitgliedern zu erfahren, wo der Schuh drückte und wie wir Unterstützung geben konnten, was uns dazu bewog, an der Mitgliederbasis eine kurze Umfrage durchzuführen. ${ }^{1}$

\section{Allgemeine Verunsicherung}

Die Tatsache, dass sich das Bundesamt für Gesundheit (BAG) immer wieder schwertat, mangels Erfahrung mit dem Virus nachvollziehbare Anweisungen zu geben, löste unter den Mitgliedern breite Verunsicherung und ab und $\mathrm{zu}$ auch gewaltigen Ärger aus. Die «Verordnung 2 über Massnahmen zur Bekämpfung des Coronavirus (COVID-19)» gab im März zunächst vor, dass Psychotherapeut*innen während den kommenden zwei Monaten nur Notfälle behandeln durften, die Praxen aber offenbleiben mussten. Mit dieser Massnahme wurde automatisch ausgeschlossen, dass die selbstständig erwerbenden Psychotherapeut*innen in den Genuss der Erwerbsersatzentschädigung kamen, die für die Zeit der COVID-19-Pandemie zügig eingerichtet worden war. Wie eine Reihe andere Selbstständigerwerbende auch, war unser Berufsstand bei dieser Vorsorgeeinrichtung offenbar schlicht vergessen worden, was viele unserer Mitglieder in eine existenzielle Krise stürzte. Indem wir mit vereinten Kräften und auf allen politischen Ebenen intervenierten, konnten wir schliesslich erreichen, dass auch selbstständig erwerbenden Psychotherapeut*innen Erwerbsersatz zugestanden wurde.

Viele Mitglieder beschäftigte zudem die Frage, ob Patient ${ }^{*}$ innen und Klient ${ }^{*}$ innen, die den Therapien aus verschiedenen Gründen plötzlich fernblieben, wieder zurückkommen würden.

1 Die anonyme Internetumfrage fand am 4. und 5. Mai 2020 in allen drei Sprachregionen statt und wurde innerhalb von einer Woche von 280 Mitgliedern beantwortet. Neben vier konkreten Fragen wurden die Mitglieder eingeladen, zusätzliche Kommentare zu ihrer Situation abzugeben.
Etliche Befragte vertraten die Ansicht, dass sich die wahren Auswirkungen erst später zeigen würden, und fragten sich, was dies wohl für ihre eigene Zukunft bedeuten könnte. Auch waren einzelne Mitglieder verunsichert, weil sie selbst zu einer Risikogruppe gehörten und sich nicht sicher waren, ob sie überhaupt arbeiten durften.

\section{Diskriminierung delegierter \\ Psychotherapie gegenüber Psychiatrie}

Ein grosses Ärgernis war, nicht zu wissen, ob telefonische oder fernmündliche Konsultationen für selbstständige Psychotherapeut*innen vergütet würden oder nicht, da dies weder auf kantonaler, noch auf Bundesebene geregelt war. Selbst auf wiederholtes Nachfragen, erhielten wir darauf keine Antwort, sodass wir uns in dieser Beziehung auch heute noch in einer Grauzone befinden.

Anders war dies für die delegierte Psychotherapie, die in Verantwortung eines Arztes oder einer Ärztin in deren Praxis durchgeführt wird. Zwar waren telefonische Konsultationen erlaubt und tarifiert. Als aber die Weisung des BAG erfolgte, dass telefonische Konsultationen während der Pandemie bzw. für sechs Monate von 240 auf 360 Minuten erhöht werden, löste dies allenthalben Kopfschütteln aus. Diese Bestimmung stiess auf einhellige Kritik nicht nur der Psychotherapeut*innen, sondern auch der Ärzteschaft. Sie sei besonders stossend, ja lächerlich, wo doch viele Patient*innen und Klient*innen aus Angst vor Ansteckung ihre Wohnungen nicht verlassen oder keine öffentlichen Verkehrsmittel benutzen wollten. Im Gegensatz dazu wurde Psychiater ${ }^{\star}$ innen praktisch unbegrenzte Zeit für fernmündliche Psychotherapien zugestanden, was grosses Unverständnis und Empörung auslöste und schlicht nicht nachvollziehbar war.

Auf unsere Forderung ans BAG, diese Ungerechtigkeit zu beheben, erhielten wir die lapidare Antwort, dass die Ausdehnung auf 360 Minuten ja eine Erhöhung sei. Dass dies pro Monat gerade mal eine Stunde telefonische Konsultation bedeutete, machte uns ratlos.

\section{«Bleiben Sie zu Hause»}

Die Aufforderung «Bleiben Sie zu Hause» und die Schliessung der Schulen stellte Eltern mit Kindern vor besonders grosse Herausforderungen. Die Situation von Familien in dieser unge- 
wohnten Situation ist oft beschrieben worden. Bei unserer Umfrage traten zwei Aspekte speziell hervor. Besonders Psychotherapeutinnen, die alleinerziehende Mütter sind, im Homeoffice arbeiteten und daneben mit ihren Kindern das Homeschooling bewältigen mussten, schilderten die Situation als Grenzerfahrung, sowohl für sie selbst als auch für ihre Kinder.

Der zweite Aspekt betrifft das eigentliche Therapieren von Kindern, was unter den eingeschränkten Verhältnissen als sehr schwierig bis unmöglich beschrieben wurde. So sei es zum Beispiel nicht möglich, Spielsachen zu desinfizieren oder besonders im Umgang mit kleineren Kindern eine Maske zu tragen. Therapien mit Kindern hätten sich deshalb verzögert oder mussten ganz abgesagt werden.

Da wir immer wieder betonten, dass Therapien möglichst nicht unterbrochen werden sollten, empfahlen wir in unserem Schutzkonzept, Kinder sollten ihre eigenen Spielsachen in die Therapie mitbringen und je nach Situation die Verwendung eines Spuckschutzes.

\section{Pensionsalter und Risikogruppen}

Einige Mitglieder im Pensionsalter schienen die COVID-19-Pandemie zum Anlass zu nehmen, ihre Praxis aufzugeben, sei es, weil sie dies sowieso schon geplant hatten, sei es, dass die Krise ihnen den willkommenen Anstoss dazu gegeben hat.

Was die wirtschaftliche Situation dieser Gruppe betrifft, äusserten sich die Befragten sehr unterschiedlich. Die Bandbreite der Rückmeldungen reichte von Mitgliedern mit ernsthaften existenziellen Ängsten, aufgrund der Zugehörigkeit zu einer Risikogruppe grosse finanzielle Einbussen in Kauf nehmen zu müssen, bis zu Aussagen, sich im Pensionsalter privilegiert zu fühlen. Denn dank Pensionskassengeld und AHV könne man die Zeit gut überstehen.

Mit dem Zugang zur Erwerbsersatzentschädigung für Selbstständigerwerbende konnten wir

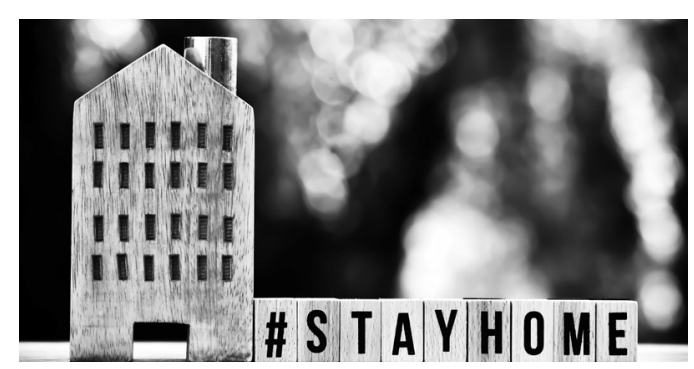

erreichen, dass auch Psychotherapeut ${ }^{*}$ innen im Pensionsalter Bezüge geltend machen konnten. Voraussetzung war, dass sie über das Pensionsalter hinaus AHV-Beiträge in die Altersvorsorge einbezahlten und über ein Einkommen verfügten, das jedoch nach unten und nach oben limitiert war.

Etliche Mitglieder berichteten, dass sie Patient*innen therapierten, die selbst zur Risikogruppe gehörten und ihr Zuhause nicht verlassen durften oder wollten. Hier boten Telefon und Videochat willkommenen Ersatz.

\section{Anwendung von Online-Tools und Telefontherapie}

Während die einen Befragten fernmündliche Therapien per Telefon oder Videochat als Segen empfanden, lehnten es andere strikt ab, wobei sich Pro und Kontra etwa die Waage hielten. Die Befürworter waren sich einig, dass Online-Therapie heutzutage geradezu eine Notwendigkeit sei. Aufseiten der Patient ${ }^{\star}$ innen gebe es sogar solche, die fernmündliche Therapien forderten, andere wiederum würden diese strikt ablehnen.

Aufseiten der Psychotherapeut ${ }^{*}$ innen wurde spürbar, dass bereits computeraffine Mitglieder sich mit der Umstellung leichttaten. Es war auch diese Gruppe, die das Bedürfnis äusserte, über die Pandemie hinaus an fernmündlichen Therapien festhalten zu wollen. Die andere Gruppe empfand es als nicht passend und als anstrengend, das Gegenüber nicht physisch vor sich zu sehen. Es ist auch diese Gruppe, die am meisten mit existenziellen Nöten kämpft. Eine Altersgrenze lässt sich daraus jedoch nicht ableiten.

Es gab Rückmeldungen, telefonische Konsultationen hätten sehr geholfen, dies sei der einzig sinnvolle Weg in dieser Situation. Allerdings wurde bemängelt, dass es keine Garantie gebe, dass die Krankenkassen solche Konsultationen übernehmen. Es gab deshalb sogar einzelne Mitglieder, die die Telefongespräche nicht abrechneten. Die Erfahrung zeigte jedoch, dass viele Krankenkassen einen kulanten Umgang mit fernmündlichen Therapien gefunden hatten.

Für uns als Verband bedeutet dies, dass wir uns überlegen wollen, wie wir diese Entwicklung fördern und unseren Mitgliedern im Online-Bereich Unterstützung geben können.

Marianne Roth ist Geschäftsleiterin der ASP 